\title{
Chloride intracellular channel 1 regulates migration and invasion in gastric cancer by triggering the ROS-mediated p38 MAPK signaling pathway
}

\author{
WEI ZHAO, MINGSHU LU and QIWEN ZHANG \\ Department of General Surgery, People's Hospital of Laiwu, Laiwu, Shandong 271100, P.R. China
}

Received December 29, 2014; Accepted September 24, 2015

DOI: $10.3892 / \mathrm{mmr} .2015 .4459$

\begin{abstract}
Chloride intracellular channel 1 (CLIC1) has been demonstrated to be overexpressed in gastric cancer, and elevated CLIC1 expression levels are markedly associated with the processes of tumor cell migration and invasion. However, the regulatory mechanism and signaling pathway underlying these processes have remained to be elucidated. The present study examined the impact of $N$-acetyl cysteine (NAC), indanyloxyacetic acid (IAA)-94 and SB203580, inhibitors of reactive oxygen species (ROS), as well as CLIC1 and p38 mitogen-activated protein kinase (MAPK) on the migration and invasion of SGC-7901 gastric cancer cells in a hypoxia-reoxygenation (H-R) microenvironment. The results demonstrated that intracellular ROS and CLIC1 levels were increased under $\mathrm{H}-\mathrm{R}$ conditions, and that functional inhibition of CLIC1 significantly decreased the H-R-elevated ROS generation and p-p38 MAPK levels in SGC-7901 cells, as well as inhibited the migration and invasion of SGC-7901 cells. In addition, the expression levels of MMP-2 and MMP-9 were inhibited by NAC, IAA-94 and SB203580. These results indicated that CLIC1 regulates gastric cancer-cell migration and invasion via the ROS-mediated p38 MAPK signaling pathway.
\end{abstract}

\section{Introduction}

Gastric cancer remains the fifth most common type of cancer, and the third leading cause of cancer-associated mortality worldwide (1). In China, gastric cancer has a five-year relative survival rate of $\sim 20 \%$ or less, due to the fact that the majority of patients are diagnosed with metastatic gastric cancer at presentation (2). The early diagnosis of individuals at a high risk of metastasis is hindered by a lack of understanding of

Correspondence to: Mr. Qiwen Zhang, Department of General Surgery, People's Hospital of Laiwu, 1 Xuehu Street, Laiwu, Shandong 271100, P.R. China

E-mail: dr_zhangqw@163.com

Key words: gastric cancer, chloride intracellular channel 1, reactive oxygen species, invasion, p38 mitogen-activated protein kinase signaling pathway the underlying molecular mechanisms. Previous studies have recognized novel biochemical functions of chloride intracellular channel (CLIC) in the regulatory mechanism of tumor cells (3-5). CLIC proteins are components or regulators of novel intracellular anion channels in mammalian cells (6). CLIC1, one of the members of CLIC, has been demonstrated to be overexpressed in gastric tumor cells, and elevated CLIC1 are markedly associated with the metastasis of lymph nodes, and lymphatic and perineural invasion $(7,8)$. In addition, downregulation in the expression of CLIC1 following transfection with CLIC1 small interfering (si)RNAs efficiently inhibits the migration and invasion of gastric cancer cells in vitro (9). However, the potential regulatory mechanism and signaling pathway underlying the migration and invasion of gastric cancer cells remains to be elucidated.

CLIC1 is also reported to be a sensor and effector in oxidative stress (10), which is characterized by the overproduction of reactive oxygen species (ROS). Emerging evidence has suggested that elevated ROS levels function as a secondary signaling molecule and are involved in the cellular processes of cancer migration and invasion $(11,12)$. Previous studies have demonstrated that intracellular ROS levels activate the mitogen-activated protein kinase (MAPK) signaling pathway (13-15), and trigger the ROS/eukaryotic protein kinase signaling pathway, which is involved in the CLIC1-mediated regulation of cell migration and invasion in colon cancer (16). Whether similar effects and mechanisms are present in gastric cancer remains to be elucidated.

It is well-known that ROS can be produced in a hypoxia and reoxygenation (H-R) microenvironment (17), and CLIL1 was reported to be involved in colon cancer metastasis under H-R conditions (18). The present study hypothesized that CLIC1 may mediate the migration and invasion of gastric cancer cells via the ROS/p38 MAPK signaling pathway. To test this hypothesis, the present study assessed the migration and invasion of SGC-7901 gastric cancer cells following downregulation of intracellular ROS levels under H-R conditions, and investigated whether this process is regulated by the ROS/p38 MAPK signaling pathway.

\section{Materials and methods}

Materials and reagents. The SGC-7901 human gastric cancer cell line was obtained from the Shanghai Institute for Biological 
Sciences of the Chinese Academy of Sciences (Shanghai, China). The specific inhibitor of ROS, N-acetyl cysteine (NAC), was purchased from Beyotime Institute of Biotechnology (Nantong, China). The inhibitor of CLIC1, indanyloxyacetic (IAA)-94 was purchased from Sigma-Aldrich (St. Louis, MO, USA). The chemical inhibitor of p38-MAPK (SB203580) was purchased from Merck Millipore (Darmstadt, Germany). Antibodies targeting p38 MAPK, phosphorylated (p)-p38 MAPK, matrix metalloproteinase (MMP)-2 and MMP-9 were purchased from Santa Cruz Biotechnology, Inc. (Dallas, TX, USA).

SGC-7901 cell culture and treatment. The SGC-7901 human gastric cancer cell line was incubated in $1 \%$ Dulbecco's modified Eagle's medium (DMEM) supplemented with 10\% fetal bovine serum (FBS; Sigma-Aldrich), $100 \mathrm{U} / \mathrm{ml}$ penicillin and $100 \mu \mathrm{g} / \mathrm{ml}$ streptomycin (both from Cosmo Bio Co., Ltd., Tokyo, Japan), and cultured at $37^{\circ} \mathrm{C}$ in an atmosphere containing $5 \% \mathrm{CO}_{2}$ with saturated humidity. $\mathrm{H}-\mathrm{R}$ conditions were produced, as previously described $(19,20)$. Briefly, for H-R treatment, the SGC-7901 cells $\left(1 \times 10^{4}\right.$ cells/well) cultured in serum-free media were placed in a humidified incubator under hypoxia $\left(95 \% \mathrm{~N}_{2}\right.$ and $5 \% \mathrm{CO}_{2}$ ) for $6 \mathrm{~h}$. Following the period of hypoxia, the cells were rapidly transferred to a humidified incubator containing $95 \%$ air and $5 \% \mathrm{CO}_{2}$ for $18 \mathrm{~h}$. In the normoxic control treatment group, the cells were maintained in an incubator with a humidified atmosphere of $95 \%$ air and $5 \% \mathrm{CO}_{2}$ for the same period of time as the H-R group.

Intracellular ROS measurement. The production of intracellular ROS was measured using a dichloro-dihydro-fluorescein diacetate (DCFH-DA) fluorescent probe (Invitrogen; Thermo Fisher Scientific, Inc., Waltham, MA, USA). The high intensity fluorescent compound, dichlorofluorescein (DCF), is formed following the passive entry of DCFH-DA into the cell to react with ROS. Briefly, the cells were trypsinized with a solution of $0.25 \%$ trypsin (containing 0.02\% EDTA; Sigma-Aldrich) and cultivated in 96 -well plates ( $1 \times 10^{4}$ cells/well), and pre-processed with NAC $(30 \mathrm{mmol} / \mathrm{l})$ or IAA-94 $(1,20$ or $40 \mu \mathrm{mol} / \mathrm{l})$ for $1 \mathrm{~h}$ to determine the effect of specific inhibitors on the production of ROS. Following treatment with H-R or normoxic conditions, the cells were washed twice with DMEM without FBS, and were then treated with $10 \mu \mathrm{M}$ DCFH-DA at $37^{\circ} \mathrm{C}$ in an atmosphere containing $5 \% \mathrm{CO}_{2}$ for $20 \mathrm{~min}$. The cells were then washed a further three times with phosphate-buffered saline (PBS) and observed under a fluorescence microscope (CK40F200; Olympus, Tokyo, Japan). The fluorescence intensity of DCFH-DA was measured using a Victor 3 microplate reader (PerkinElmer, Inc., Waltham, MA, USA). The cells were dissolved using 1\% SDS (Bio-Rad Laboratories, Hercules, CA, USA) and centrifuged at $14,000 \times \mathrm{g}$ for $5 \mathrm{~min}$ at $4^{\circ} \mathrm{C}$, prior to the removal of the supernatant and plating into a 96 -well plate. Measurements were made at an excitation wavelength of $485 \mathrm{~nm}$ and emission wavelength of $530 \mathrm{~nm}$. Values are expressed as the fold change, compared with the normoxic group.

Wound healing assay. A wound healing assay was performed to examine the capacity of cell migration. Following trypsinization with a solution of $0.25 \%$ trypsin and EDTA, SGC-7901 cells in the logarithmic growth phase were cultivated in 6-well plates at a density of $5 \times 10^{4}$ cells/well. When the cells grew to
$80 \%$ confluence, the cell monolayers were scratched using a sterile pipette tip to form a wound. The cells were subsequently treated with NAC (30 mmol/l), IAA-94 (40 $\mu \mathrm{mol} / \mathrm{l})$, or SB203580 ( $10 \mu \mathrm{mol} / \mathrm{l})$ for $1 \mathrm{~h}$, and then exposed to H-R or normoxic conditions for $24 \mathrm{~h}$ at $37^{\circ} \mathrm{C}$. Images of the wound healing process were captured under a microscope (Nikon Eclipse TS 100; Nikon Corporation, Tokyo, Japan) at 0 and $24 \mathrm{~h}$, respectively. The wound recovery rates (\%) were calculated using ImagePro Plus 6.0 software (Media Cybernetics, Inc., Rockville, MD, USA) and were used to indicate the migration of the SGC-7901 cells. The experiments were performed triplicate.

Cell invasion assay. SGC-7901 cell invasiveness was assayed using Biocoat Matrigel Invasion Chambers (BD Biosciences, Franklin Lakes, NJ, USA), according to the manufacturer's protocol. Briefly, the cells were trypsinized and plated in the upper chamber at a density of $1 \times 10^{5}$ cells $/ \mathrm{ml}$, and 5\% FBS was added to the lower chamber to act as a chemoattractant. To determine the effect of specific inhibitors, the cells were pre-treated with NAC (30 mmol/l), IAA-94 $(40 \mu \mathrm{mol} / \mathrm{l})$ or SB203580 $(10 \mu \mathrm{mol} / \mathrm{l})$ for $1 \mathrm{~h}$ prior to their addition to the chamber. Following exposure to H-R or normoxic conditions, the non-invading cells were removed by wiping with a cotton swab, and the invaded cells, located on the lower surface of the membrane, were fixed with paraformaldehyde (Bio-Rad Laboratories, Inc.) and stained with crystal violet (Bio-Rad Laboratories, Inc.). For quantification, the cells were counted under a microscope (X41; Olympus) in five randomly-selected fields. Data are expressed as the relative number of invaded cells, compared with those in normoxic conditions.

Western blot analysis. SGC-7901 cells were cultured until sub-confluence and protein was extracted using a mammalian cell lysis kit (Bio Basic Inc., Markham, OT, Canada). In brief, cells were collected and washed twice by cold PBS, and lysed in $50 \mu \mathrm{l}$ lysis buffer $(2 \mathrm{mmol} / \mathrm{l}$ Tris- $\mathrm{HCl} \mathrm{pH} \mathrm{7.4,} 50 \mathrm{mmol} / \mathrm{l}$ $\mathrm{NaCl}, 25 \mathrm{mmol} / 1$ EDTA, $50 \mathrm{mmol} / \mathrm{l} \mathrm{NaF}, 1.5 \mathrm{mmol} / \mathrm{l}$ $\mathrm{Na}_{3} \mathrm{VO}_{4}, 1 \%$ Triton X-100, $0.1 \%$ SDS supplemented with protease inhibitors, $1 \mathrm{mmol} / 1$ phenylmethylsulfonylfluoride, $10 \mathrm{mg} / 1$ pepstatin, $10 \mathrm{mg} / 1$ aprotinin and $5 \mathrm{mg} / 1$ leupeptin; all from Sigma-Aldrich). Then cells were centrifuged at $13,000 \times \mathrm{g}$ for $15 \mathrm{~min}$ at $4^{\circ} \mathrm{C}$, prior to collection of the supernatant. Protein concentration was measured using a Bicinchoninic Acid Protein Assay kit (Sigma-Aldrich). The proteins were then separated by $10 \%$ SDS-PAGE (Bio-Rad Laboratories, Inc.) and electrotransferred onto immunoblot nitrocellulose membranes (EMD Millipore, Billerica, MA, USA). The membranes were blocked in 3\% Tris-buffered saline containing $0.05 \%$ Tween- 20 and $5 \%$ fat-free dry milk, prior to incubation with primary antibodies overnight at $4{ }^{\circ} \mathrm{C}$, followed by horseradish peroxidase-conjugated secondary antibody for $1 \mathrm{~h}$. Primary antibodies against CLIC1 (1:400), MAPK (1:400), p-MAPK (1:400), MMP-2 (1:200), MMP-9 $(1: 200)$ and GAPDH $(1: 1,000)$ were used. Western blots were visualized by enhanced chemiluminescence reagent (GE Healthcare Bio-Sciences, Pittsburgh, PA, USA) and the density of the bands was quantified using Quantity One software (version 4.2.2; Bio-Rad Laboratories, Inc.). Experiments were performed three times and band intensities were normalized to GAPDH. 
A

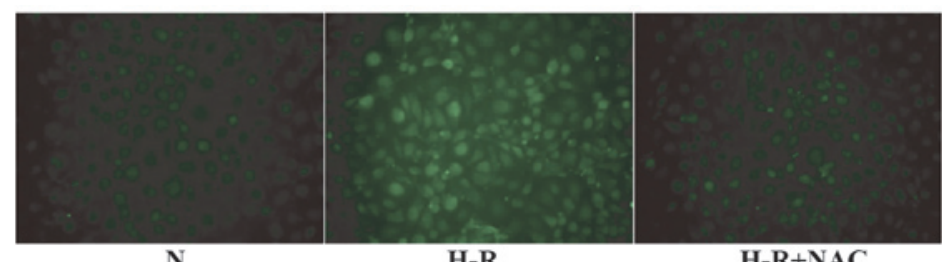

$\mathbf{N}$

H-R

H-R+NAC

B

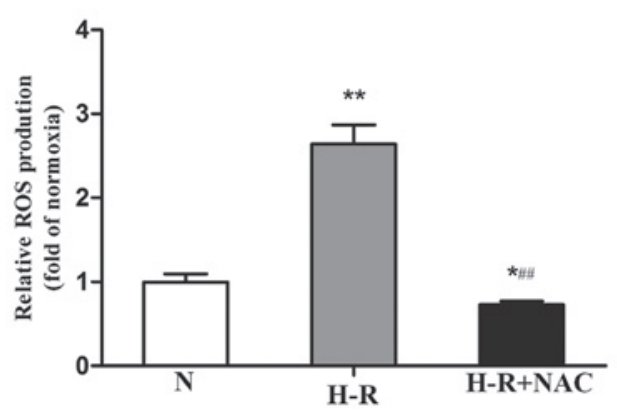

Figure 1. H-R conditions induce the production of ROS. (A) SGC-7901 human gastric cancer cells were pre-treated with the antioxidant NAC (30 mmol/l) for $1 \mathrm{~h}$ prior to exposure to H-R conditions for $24 \mathrm{~h}$. ROS production was analyzed using DCFH-DA staining under a fluoresence microscope (magnification, $\mathrm{x} 40$ ), DCF fluorescence was markedly increased under H-R conditions, and after exposure to the NAC anti-oxidant, the DCF fluorescence was significantly decreased compared with that in the H-R group. (B) Fluorescence intensity of DCFH-DA was measured using a Victor 3 microplate reader. Values are expressed as the fold of normoxia of three independent experiments and are presented as the mean \pm standard deviation. ${ }^{*} \mathrm{P}<0.05$ and ${ }^{* * *} \mathrm{P}<0.01$, vs, normoxic group; ${ }^{\# \#} \mathrm{P}<0.01$, vs. H-R group. N, normoxia; H-R, hypoxia-reoxygenation; ROS, reactive oxygen species; NAC, N-acetyl cysteine; DCFH-DA, dichloro-dihydro-fluorescein diacetate.

Statistical analysis. All data are presented as the mean \pm standard deviation of three experiments. Statistical analysis was performed using SPSS software, version 13.0 (SPSS, Inc., Chicago, IL, USA). Statistical comparisons were performed using one-way analysis of variance. $\mathrm{P}<0.05$ between the H-R group and control group was considered to indicated a statistically significant difference.

\section{Results}

$H-R$ conditions induce intracellular ROS production in SGC-7901 gastric cancer cells. To determine whether H-R conditions induced the production of ROS, an intracellular ROS-sensitive fluorescence indicator, DCFH-DA, was used. The oxidation of non-fluorescent DCFH to highly fluorescent DCF, which emits green fluorescence, provides a quantitative assay of ROS generation (21). Following exposure to H-R conditions, $\mathrm{DCF}^{+}$fluorescence was markedly increased (Fig. 1A), and was 2.64 times higher than that in the normoxic group $(\mathrm{F}=11.63$; $\mathrm{P}<0.001$; Fig. $1 \mathrm{~B})$, indicating that $\mathrm{H}-\mathrm{R}$ conditions induced the production of ROS in the SGC-7901 gastric cancer cells. Following exposure to the NAC antioxidant, the levels of ROS in the SGC-7901 gastric cancer cells were 0.73-fold of the normoxic group and were significantly decreased, compared with the $\mathrm{H}-\mathrm{R}$ group $(\mathrm{F}=14.48 ; \mathrm{P}<0.001)$. These results suggested that the levels of intracellular ROS in SGC-7901 gastric cancer cells increased under H-R conditions.

Functional inhibition of CLIC1 downregulates ROS production in SGC-7901 gastric cancer cells. As CLIC1 may function as a 'sensor' and 'effector' of the oxidative stress in cells (10), a specific CLIC1 inhibitor, IAA-94, was introduced into the SGC-7901 gastric cancer cells to further determine the factors involved in the production of ROS. Following incubation with
IAA-94 at different concentrations (1, 20 and $40 \mu \mathrm{mol} / \mathrm{l}), \mathrm{ROS}$ generation was reduced in a concentration-dependent manner. IAA-94 (20 and $40 \mu \mathrm{mol} / \mathrm{l})$ induced marked inhibition of ROS, compared with the H-R group $(\mathrm{F}=13.59 ; \mathrm{P}<0.001 ; \mathrm{F}=15.14$; $\mathrm{P}<0.001$ ), as shown in Fig. 2A. Western blot analysis revealed that the protein expression levels of CLIC1 in the SGC-7901 gastric cancer cells under H-R conditions were significantly increased, compared with those in the normoxic control group, $(\mathrm{F}=4.658 ; \mathrm{P}=0.009)$. However, IAA-94 did not downregulate the elevated protein expression levels of CLIC1 (Fig. 2B and C). These results suggested that the functional inhibition of CLIC1 may be involved in downregulating the production of ROS in SGC-7901 gastric cancer cells.

CLICl is involved in the regulation of SGC-7901 gastric cancer cell migration. The metastatic process of cancer cells is usually accompanied by morphological changes, to enable the cells to pass through narrow extracellular spaces in order to metastasize (22). In the present study, a wound healing assay was performed to examine the capacity of cell migration. As shown in Fig. 3, compared with the normoxic control group, $\mathrm{H}-\mathrm{R}$ conditions caused a significant increase in wound recovery $(\mathrm{F}=6.745 ; \mathrm{P}=0.003)$. In addition, NAC and IAA-94 $(40 \mu \mathrm{mol} / \mathrm{l})$ inhibited this wound healing effect. These results suggested that CLICl was involved in regulating the migration of SGC-7901 gastric cancer cells, and this effect may be achieved via the ROS-mediated signaling pathway. As p38 MAPK has been demonstrated to be an important signaling molecule for cell invasion and migration in human breast epithelial cells (23), $10 \mu \mathrm{mol} / 1 \mathrm{SB} 203580$, a chemical inhibitor of $\mathrm{p} 38$, was used to determine whether the ROS/p38 MAPK signaling pathway contributed to the migration of the SGC-7901 gastric cancer cells. SB203580 also inhibited the elevated cell motility induced by H-R conditions, indicating that the ROS/p38 MAPK 


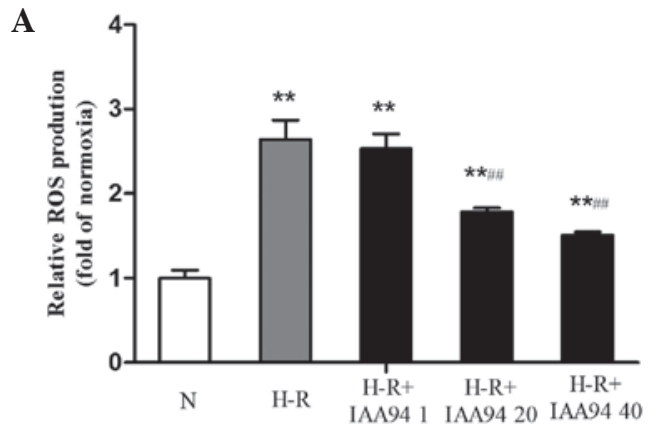

B
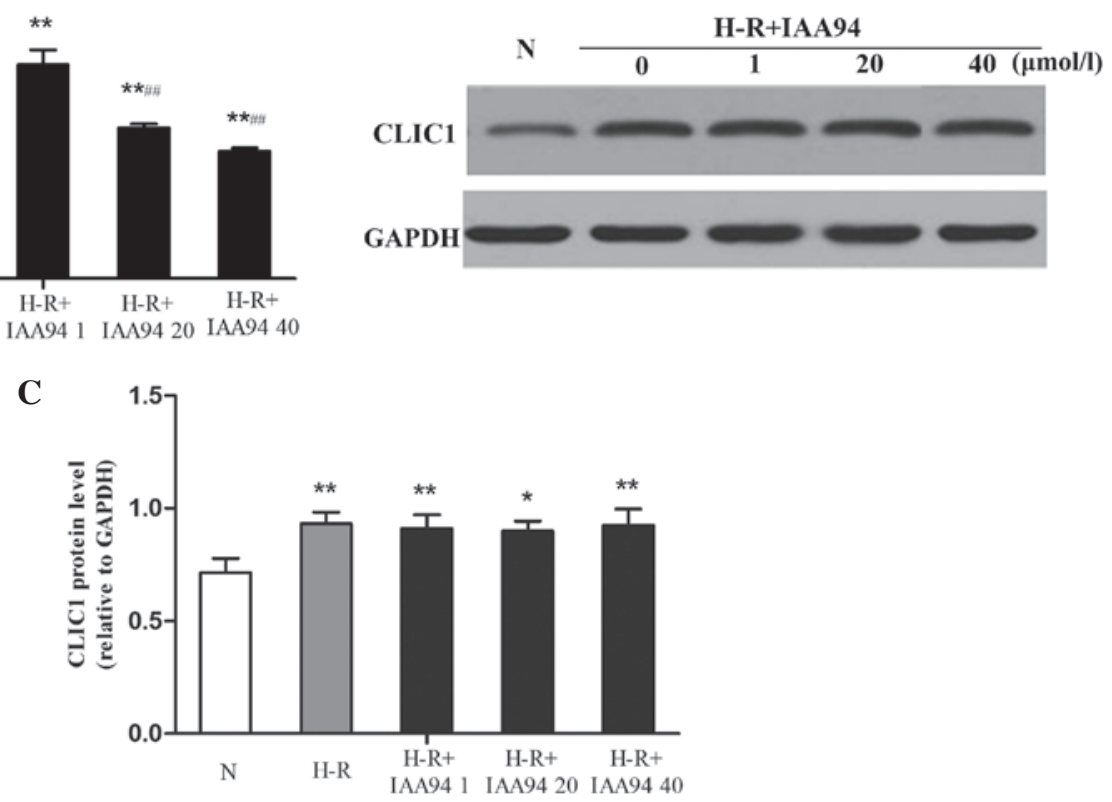

Figure 2. CLIC1 regulates the production of ROS in SGC-7901 gastric cancer cells under H-R conditions. SGC-7901 cells were pre-treated with the CLIC1 inhibitor, IAA-94 (1, 20 and $40 \mu \mathrm{mol} / \mathrm{l})$, for $1 \mathrm{~h}$ followed by H-R condition exposure for $24 \mathrm{~h}$. ROS production was analyzed using DCFH-DA staining and the fluorescence intensity of DCFH-DA was measured using a Victor 3 microplate reader. (A) Data are expressed as the fold of normoxia. (B and C) Protein expression levels of CLIC1 were measured using western blot analysis, with band intensities were normalized to GAPDH. Data are presented as the mean \pm standard deviation of three independent experiments. " $\mathrm{P}<0.05$ and ${ }^{* *} \mathrm{P}<0.01$, vs, normoxic group; ${ }^{\# /} \mathrm{P}<0.01$, vs. H-R group. N, normoxia; H-R. H-R, hypoxia-reoxygenation; ROS, reactive oxygen species; DCFH-DA, dichloro-dihydro-fluorescein diacetate; IAA-94, indanyloxyacetic acid 94; CLIC1, chloride intracellular channel 1.

A

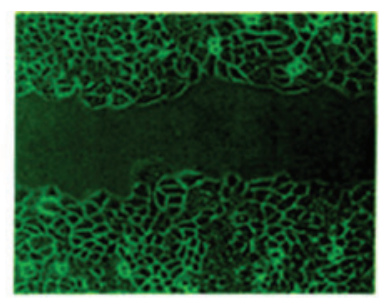

N

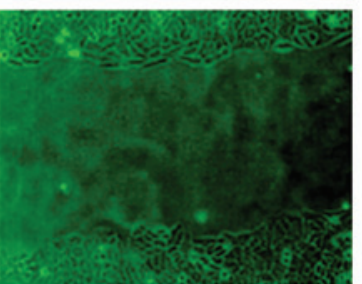

H-R+NAC

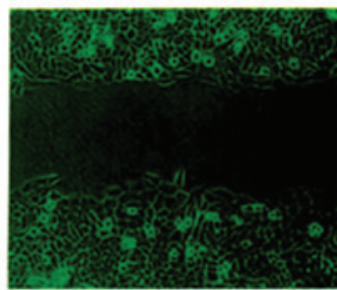

H-R+IAA94

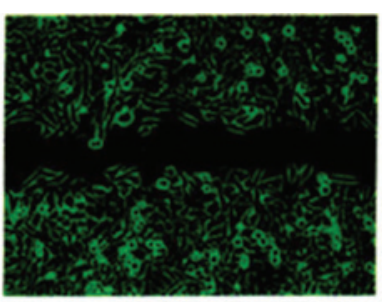

H-R

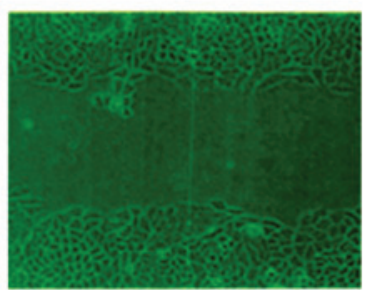

H-R+SB204580
B

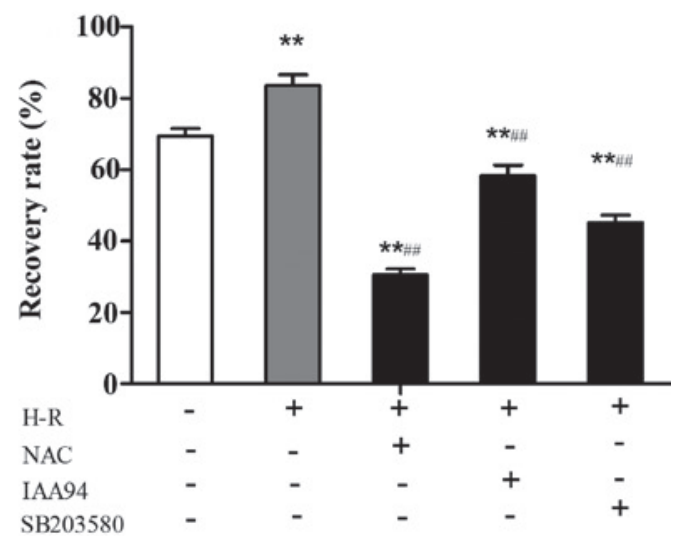

SB203580

Figure 3. CLICl is involved in the regulation SGC-7901 gastric cancer cell migration. A wound healing assay was performed to H-R or normoxic conditions for $24 \mathrm{~h}$. (A) Images of the wound healing process were captured using a microscope (magnification, x100). (B) Wound recovery rates (\%) were quantified using ImageProPlus 6.0 software, and presented as the mean \pm standard deviation of three experiments. ${ }^{* *} \mathrm{P}<0.01$, vs, normoxic group; ${ }^{\# \#} \mathrm{P}<0.01$, vs. $\mathrm{H}-\mathrm{R}$ group. N, normoxia; H-R. H-R, hypoxia-reoxygenation; IAA-94, indanyloxyacetic acid 94; NAC, N-acetyl cysteine.

signaling pathway is involved in the migration of SGC-7901 gastric cancer cells.

CLICl is involved in the regulation of SGC-7901 gastric cancer cell invasion. The effect of CLIC1 on SGC-7901 gastric cancer cell invasiveness was examined using Matrigel invasion assays in vitro. As shown in Fig. 4, compared with the normoxic control group, the invasiveness of the SGC-7901 gastric cancer cells was markedly increased under
H-R conditions $(\mathrm{F}=15.063 ; \mathrm{P}<0.001)$. Following pre-treatment with NAC $(30 \mathrm{mmol} / \mathrm{l})$ or IAA-94 $(40 \mu \mathrm{mol} / \mathrm{l})$, the elevated invasiveness caused by H-R exposure was inhibited. Treatment with the SB203580 (10 $\mu \mathrm{mol} / \mathrm{l})$ inhibitor of p38 MAPK also inhibited the elevated cell invasiveness induced by H-R conditions. These results suggested that CLICl was involved in the regulation of SGC-7901 gastric cancer cell invasion, and this process may activate the ROS-p38 MAPK signaling pathway. 
A
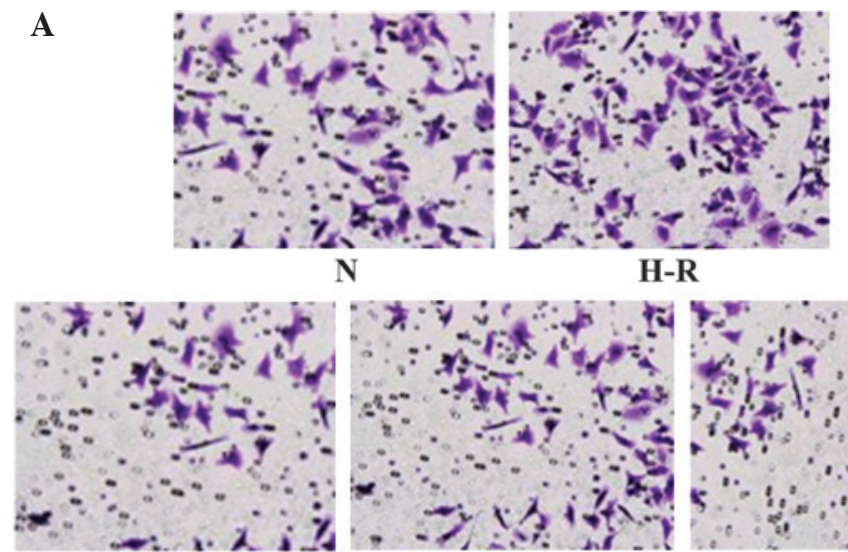

H-R+NAC

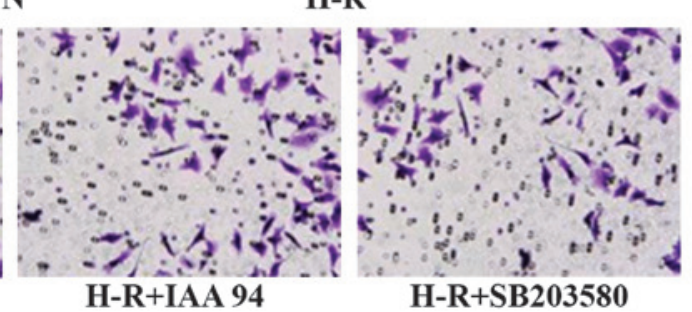

B

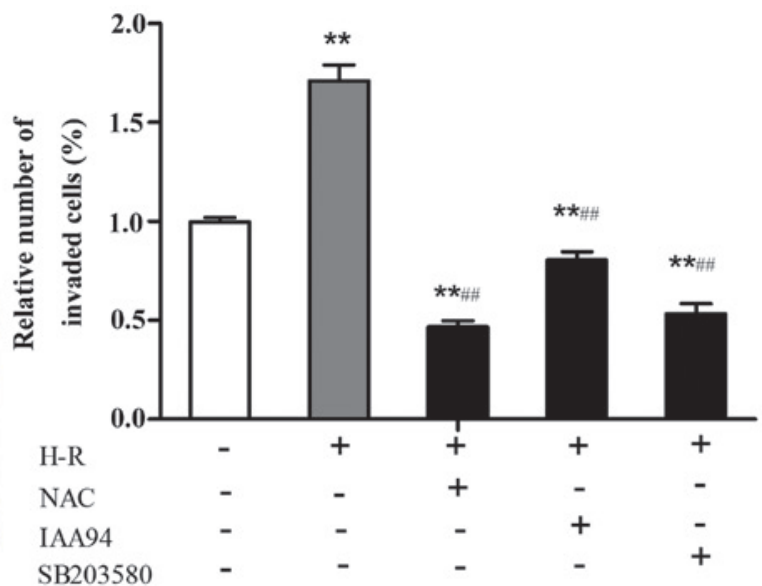

Figure 4. CLICl is involved in the regulation of SGC-7901 gastric cancer cell invasion. Cells were treated with NAC (30 mmol/1), IAA-94 (40 $\mu$ mol/1) or SB203580 $(10 \mu \mathrm{mol} / \mathrm{l})$ for $1 \mathrm{~h}$ and then exposed to $\mathrm{H}-\mathrm{R}$ or normoxic conditions for $24 \mathrm{~h}$. A Matrigel invasion assay was performed to examine SGC-7901 gastric cancer cell invasiveness. (A) Invaded cells were fixed and stained with crystal violet and observed under a microscope (magnification, x100). (B) Numbers of invaded ceslls were quantified under a microscope in five randomly-selected fields. Data are expressed as the relative number of invading cells compared with those under normoxic conditions and are presented as the mean \pm standard deviation. ${ }^{* *} \mathrm{P}<0.01$, vs, normoxic group; ${ }^{\# \#} \mathrm{P}<0.01$, vs. H-R group. $\mathrm{N}$, normoxia; H-R H-R, hypoxia-reoxygenation; IAA-94, indanyloxyacetic acid 94; NAC, N-acetyl cysteine.

$\mathbf{A}$
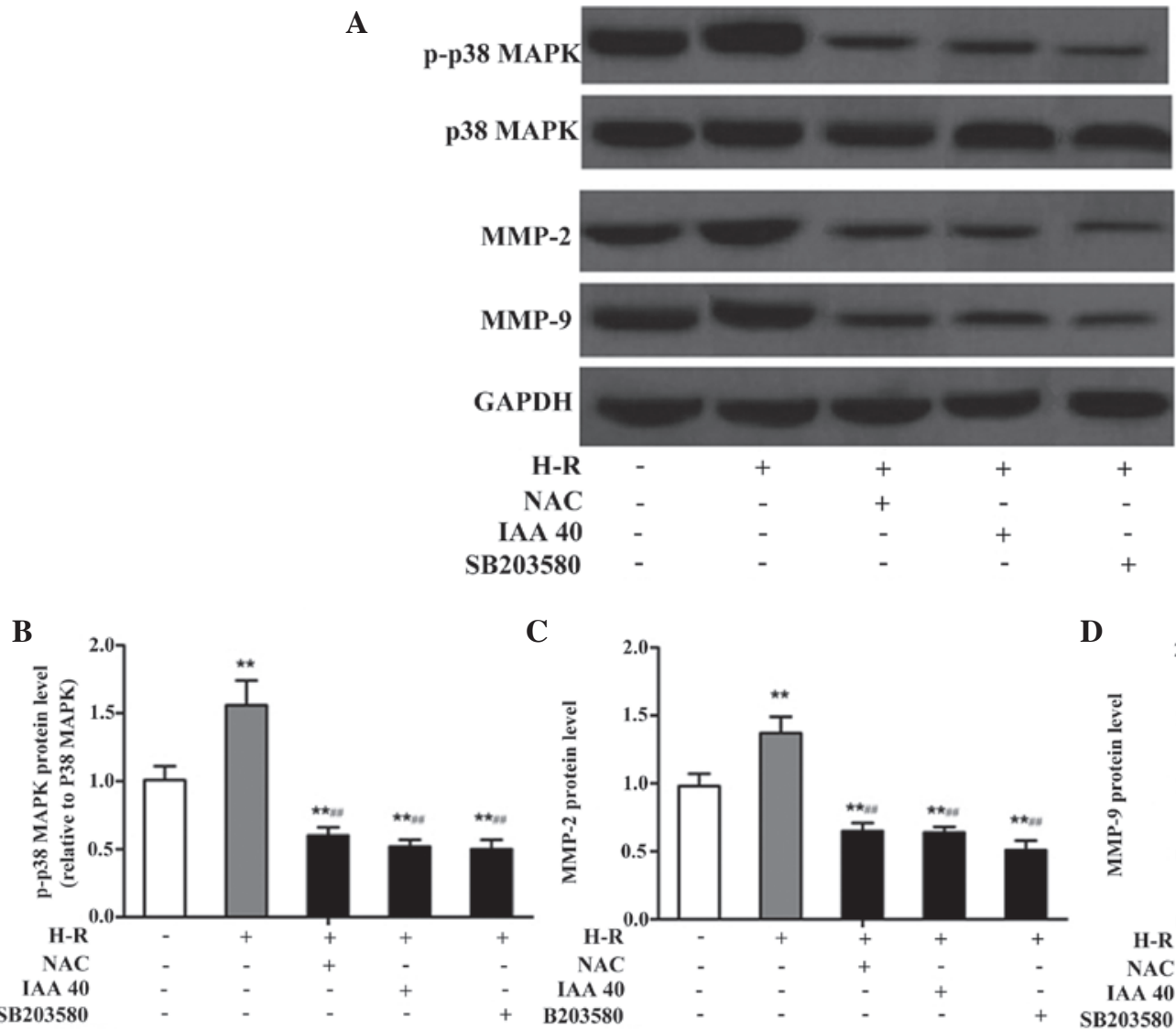

C

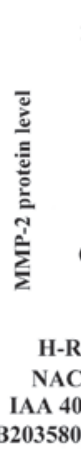

D

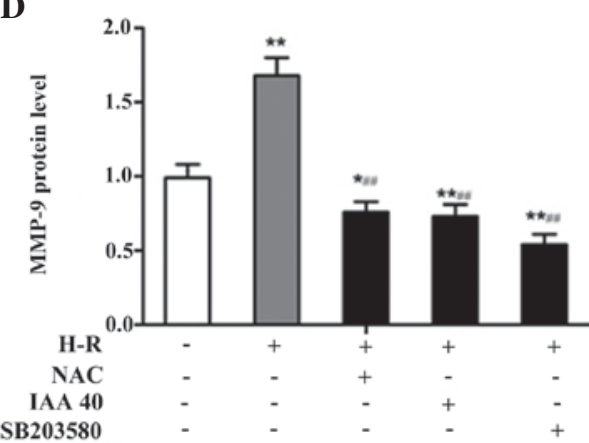

Figure 5. CLICl regulates gastric cancer cell migration and invasion via the ROS-mediated p38 MAPK signaling pathway. The cells were treated with NAC (30 mmol/l), IAA-94 $(40 \mu \mathrm{mol} / \mathrm{l})$ or SB203580 $(10 \mu \mathrm{mol} / \mathrm{l})$ for $1 \mathrm{~h}$, and then exposed to H-R or normoxic conditions for $24 \mathrm{~h}$. (A) Protein expression levels of p-p 38 MAPK (relative to p38 MAPK), MMP-2 and MMP-9 (normalized to GAPDH) were measured using western blot analysis. (B-D) Quantified band intensities are presented as the mean \pm standard deviation of three independent experiments. ${ }^{*} \mathrm{P}<0.05$ and ${ }^{* *} \mathrm{P}<0.01$, vs, normoxic group; ${ }^{\# \#} \mathrm{P}<0.01$, vs. H-R group. ROS, reactive oxygen species; p, phospho; MAPK, mitogen-activated protein kinase; N, normoxia; H-R, hypoxia-reoxygenation; IAA-94, indanyloxyacetic acid 94; NAC, N-acetyl cysteine; MMP, matrix metalloproteinase.

CLICl regulates gastric cancer cell migration and invasion via the ROS-mediated 38 MAPK signaling pathway. It has been demonstrated that the p38 MAPK signaling pathway is associated with cancer migration. In order to further examine whether $\mathrm{CLICl}$ regulates gastric cancer cell migration and invasion via the ROS-mediated p38 MAPK signaling pathway, the 
expression levels of p-p38 MAPK were examined. In addition, the activities of MMP-2 and MMP-9, two important mediators of cancer metastasis were quantified using western blotting. H-R exposure led to a significant increase in the expression levels of p-p38, MMP-2 and MMP-9, and these effects were be inhibited by treatment with NAC, IAA-94 and SB203580 (Fig. 5A). Compared with the normoxic group, the protein expression levels of $\mathrm{p}-\mathrm{p} 38(\mathrm{~F}=4.626 ; \mathrm{P}=0.009)$, MMP-2 $(\mathrm{F}=4.503 ; \mathrm{P}=0.011)$ and MMP-9 ( $\mathrm{F}=7.967 ; \mathrm{P}=0.001)$ were significantly increased following H-R exposure (Fig. 5B-D). By contrast, the protein expression levels of $\mathrm{p}-\mathrm{p} 38$ ( $\mathrm{F}=8.764 ; \mathrm{P}<0.001)$, MMP-2 ( $\mathrm{F}=5.284$; $\mathrm{P}=0.006)$ and MMP-9 $(\mathrm{F}=11.476 ; \mathrm{P}<0.001)$ were significantly reduced following treatment with NAC, compared with the H-R treatment group, which indicated that ROS activated the p38 MAPK signaling pathway under H-R conditions. Similar effects were observed following treatment with IAA-94 and SB203580. These results suggested that CLIC1 regulated gastric cancer cell metastasis, which was activated via the ROS-mediated p38 MAPK signaling pathway.

\section{Discussion}

Metastasis of cancer cells is a complex biological process, which is associated with changes in specific cytokines and signaling pathways $(24,25)$. Tumor hypoxia promotes the malignant tumor cell phenotype, and is one of the mechanisms underlying increased tumor aggressiveness (26). Previously, studies have demonstrated that tumor cells exist in the H-R environment due to its irregular microvascular network and blood flow patterns $(27,28)$. Based on these biological hypotheses, the present study exposed gastric cancer cells to H-R treatment, and the results demonstrated that the migration and invasion of gastric cancer cells were significantly increased under H-R conditions. These findings were concordant with those of previous studies, in which H-R was demonstrated to markedly regulate cell migration in breast cancer (29) and cell invasiveness in pancreatic cancer (19).

CLIC1 is markedly associated with the migration and invasion of gastric cancer, which is supported by evidence presented in previous studies. Chen et al (8) reported that the expression levels of CLIC1 in tumor regions increased 1.95 -fold, compared with adjacent non-cancerous tissue samples, and elevated CLIC1 was associated with lymph node metastasis, lymphatic and perineural invasion and pathological staging. Ma et al (9) revealed that transfection of the SGC-7901 gastric cancer cell line with CLIC1 siRNA effectively downregulated the protein expression levels of CLIC1, which led to the inhibition of invasion and migration by 54.32 and $29.26 \%$, respectively. However, the molecular mechanisms underlying these processes remain to be elucidated. CLIC1 may act as a 'sensor' and 'effector' of the process of oxidative stress (10), in which CLIC1 reacts to the transformation of the membrane, resulting in its overexpression and the enhancement of channel activity. Based on these findings, the present study further investigated whether the expression of $\mathrm{CLICl}$ was associated with oxidative stress in gastric cancer. The results demonstrated that $\mathrm{H}-\mathrm{R}$ conditions induced a marked increase in the expression levels of CLIC1 and ROS. Although the inhibitor of CLIC1, IAA-94, did not downregulate the elevated protein expression levels of CLIC1, the H-R-induced elevation in intracellular ROS levels were significantly inhibited by IAA-94, suggesting that the functional inhibition of the activity of the CLIC1 signaling pathway may be involved in downregulating ROS production in SGC-7901 gastric cancer cells. Therefore, CLICl was involved in the metastasis and invasion of gastric cancer cells, and these processes were produced through the regulation of intracellular ROS.

ROS are constantly generated and eliminated to maintain equilibrium under biological conditions, and are associated with the regulation of various physiological and pathological processes, including cell differentiation, proliferation and apoptosis $(30,31)$. Previous studies have suggested that ROS and their associated redox-sensitive signaling pathways may be involved in tumor metastasis (32-35). Chronic and sustained generation of ROS can activate certain metastasis-associated proteins, including MMPs, which are regulated by MAPK signal transduction pathways (36-38). The p38 MAPK signaling pathway has been identified as an important member of the MAPK family. It is activated by environmental and genotoxic stresses, and is involved, not only in the regulation of cell growth, differentiation, death and synchronization between cellular function, but also in the proliferation, differentiation, migration and invasion of specific cell types with positive regulation $(39,40)$. MMPs can affect the degradation of the extracellular matrix (ECM), permitting cancer cells to migrate across the basal membrane prior to invading adjacent or distant tissues and organs (41). As MMP-2 and MMP-9 can degrade the majority of ECM components forming the basal membrane, the expression levels of MMP-2 and MMP-9, as well as p-p38 MAPK were examined in the present study using western blotting. The results demonstrated that exposure to H-R conditions led to a significant increase in the expression levels of p-p38, MMP-2 and MMP-9, and this increase was inhibited by NAC, IAA-94 and SB203580, indicating that the ROS-mediated p38 MAPK signaling pathway was involved in CLIC1-regulated gastric cancer cell metastasis.

In conclusion, the involvement of the ROS-mediated p38 MAPK signaling pathway in SGC-7901 cell metastasis was further supported by investigations in the present study using ROS, CLIC1 and p38 MAPK inhibitors, which demonstrated that CLIC1 activated the p38 MAPK signaling pathway during gastric cancer cell migration and invasion, and this process was mediated by ROS. Although the use of SGC-7901 cell lines as a model system to investigate gastric cancer metastasis may not represent the tumor in its entirety, the information provided in the present study contributes to an improved understanding of the molecular mechanisms underlying CLIC1-regulated gastric cancer metastasis.

\section{References}

1. Fock KM: Review article: The epidemiology and prevention of gastric cancer. Aliment Pharmacol Ther 40: 250-260, 2014

2. Lin Y, Ueda J, Kikuchi S, Totsuka Y, Wei WQ, Qiao YL and Inoue M: Comparative epidemiology of gastric cancer between Japan and China. World J Gastroenterol 17: 4421-4428, 2011.

3. Ko JH, Ko EA, Gu W, Lim I, Bang H and Zhou T: Expression profiling of ion channel genes predicts clinical outcome in breast cancer. Mol Cancer 12: 106, 2013.

4. Poulsen KA, Andersen EC, Hansen CF, Klausen TK, Hougaard C, Lambert IH and Hoffmann EK: Deregulation of apoptotic volume decrease and ionic movements in multidrug-resistant tumor cells: Role of chloride channels. Am J Physiol Cell Physiol 298: C14-C25, 2010. 
5. Shiozaki A, Otsuji E and Marunaka Y: Intracellular chloride regulates the $\mathrm{G}(1) / \mathrm{S}$ cell cycle progression in gastric cancer cells. World J Gastrointest Oncol 3: 119-122, 2011.

6. Ashley RH: Challenging accepted ion channel biology: p64 and the CLIC family of putative intracellular anion channel proteins (Review). Mol Membr Biol 20: 1-11, 2003.

7. Zheng DL, Huang QL, Zhou F, Huang QJ, Lin JY and Lin X: PA28 $\beta$ regulates cell invasion of gastric cancer via modulating the expression of chloride intracellular channel 1. J Cell Biochem 113: 1537-1546, 2012.

8. Chen CD, Wang CS, Huang YH, Chien KY, Liang Y, Chen WJ and Lin KH: Overexpression of CLIC1 in human gastric carcinoma and its clinicopathological significance. Proteomics 7: 155-167, 2007.

9. Ma PF, Chen JQ, Wang Z, Liu JL and Li BP: Function of chloride intracellular channel 1 in gastric cancer cells. World J Gastroenterol 18: 3070-3080, 2012.

10. Averaimo S, Milton RH, Duchen MR and Mazzanti M: Chloride intracellular channel 1 (CLIC1): Sensor and effector during oxidative stress. FEBS Lett 584: 2076-2084, 2010.

11. Costa A, Scholer-Dahirel A and Mechta-Grigoriou F: The role of reactive oxygen species and metabolism on cancer cells and their microenvironment. Semin Cancer Biol 25: 23-32, 2014.

12. Okoh V, Deoraj A and Roy D: Estrogen-induced reactive oxygen species-mediated signalings contribute to breast cancer. Biochim Biophys Acta 1815: 115-133, 2011.

13. Son Y, Kim S, Chung HT and Pae HO: Reactive oxygen species in the activation of MAP kinases. Methods Enzymol 528: 27-48, 2013.

14. Son Y, Cheong YK, Kim NH, Chung HT, Kang DG and Pae HO: Mitogen-Activated Protein Kinases and Reactive Oxygen Species: How Can ROS Activate MAPK Pathways? J Signal Transduct 2011: 792639, 2011.

15. Tormos AM, Taléns-Visconti R, Nebreda AR and Sastre J: p38 MAPK: A dual role in hepatocyte proliferation through reactive oxygen species. Free Radic Res 47: 905-916, 2013.

16. Wang P, Zeng Y, Liu T, Zhang C, Yu PW, Hao YX, Luo HX and Liu G: Chloride intracellular channel 1 regulates colon cancer cell migration and invasion through ROS/ERK pathway. World J Gastroenterol 20: 2071-2078, 2014.

17. Li C and Jackson RM: Reactive species mechanisms of cellular hypoxia-reoxygenation injury. Am J Physiol Cell Physiol 282: C227-C241, 2002.

18. Wang P, Zhang C, Yu P, Tang B, Liu T, Cui H and Xu J: Regulation of colon cancer cell migration and invasion by CLIC1-mediated RVD. Mol Cell Biochem 365: 313-321, 2012.

19. Binker MG, Binker-Cosen AA, Richards D, Gaisano HY, de Cosen RH and Cosen-Binker LI: Hypoxia-reoxygenation increase invasiveness of PANC-1 cells through Rac1/MMP-2. Biochem Biophys Res Commun 393: 371-376, 2010.

20. Kokura S, Yoshida N, Imamoto E, Ueda M, Ishikawa T, Uchiyama K, Kuchide M, Naito Y, Okanoue T and Yoshikawa T: Anoxia/reoxygenation down-regulates the expression of E-cadherin in human colon cancer cell lines. Cancer Lett 211 79-87, 2004.

21. Su HL, Chou CC, Hung DJ, et al: The disruption of bacterial membrane integrity through ROS generation induced by nanohybrids of silver and clay. Biomaterials 30: 5979-5987, 2009.

22. Chaffer CL and Weinberg RA: A perspective on cancer cell metastasis. Science 331: 1559-1564, 2011.

23. Kim MS, Lee EJ, Kim HR and Moon A: p38 kinase is a key signaling molecule for H-Ras-induced cell motility and invasive phenotype in human breast epithelial cells. Cancer Res 63: 5454-5461, 2003.
24. Negus RP and Balkwill FR: Cytokines in tumour growth, migration and metastasis. World J Urol 14: 157-165, 1996.

25. Versteeg HH, Spek CA, Peppelenbosch MP and Richel DJ: Tissue factor and cancer metastasis: The role of intracellular and extracellular signaling pathways. Mol Med 10: 6-11, 2004.

26. Avni R, Cohen B and Neeman M: Hypoxic stress and cancer: Imaging the axis of evil in tumor metastasis. NMR Biomed 24: 569-581, 2011.

27. Bendinelli P, Maroni P, Matteucci E, Luzzati A, Perrucchini G and Desiderio MA: Microenvironmental stimuli affect Endothelin-1 signaling responsible for invasiveness and osteomimicry of bone metastasis from breast cancer. Biochim Biophys Acta 1843: 815-826, 2014.

28. Quail DF and Joyce JA: Microenvironmental regulation of tumor progression and metastasis. Nat Med 19: 1423-1437, 2013.

29. Postovit LM, Abbott DE, Payne SL, Wheaton WW, Margaryan NV, Sullivan R, Jansen MK, Csiszar K, Hendrix MJ and Kirschmann DA: Hypoxia/reoxygenation: A dynamic regulator of lysyl oxidase-facilitated breast cancer migration. J Cell Biochem 103: 1369-1378, 2008.

30. Circu Ml and Aw TY: Reactive oxygen species, cellular redox systems and apoptosis. Free Radic Biol Med 48: 749-762, 2010

31. Kamata H, Honda S, Maeda S, Chang L, Hirata $\mathrm{H}$ and Karin M: Reactive oxygen species promote TNFalpha-induced death and sustained JNK activation by inhibiting MAP kinase phosphatases. Cell 120: 649-661, 2005.

32. Inokuma T, Haraguchi M, Fujita F, Torashima Y, Eguchi S and Kanematsu T: Suppression of reactive oxygen species develops lymph node metastasis in colorectal cancer. Hepatogastroenterology 59: 2480-2483, 2012.

33. Nishikawa M: Reactive oxygen species in tumor metastasis. Cancer lett 266: 53-59, 2008.

34. Arnold RS, Shi J, Murad E, Whalen AM, Sun CQ, Polavarapu R, Parthasarathy S, Petros JA and Lambeth JD: Hydrogen peroxide mediates the cell growth and transformation caused by the mitogenic oxidase Nox1. Proc Natl Acad Sci USA 98: 5550-5555, 2001

35. Allen RG and Tresini M: Oxidative stress and gene regulation. Free Radic Biol Med 28: 463-499, 2000.

36. Chen PN, Hsieh YS, Chiou HL and Chu SC: Silibinin inhibits cell invasion through inactivation of both PI3K-Akt and MAPK signaling pathways. Chem Biol Interact 156: 141-150, 2005.

37. Kwon GT, Cho HJ, Chung WY, Park KK, Moon A and Park JH: Isoliquiritigenin inhibits migration and invasion of prostate cancer cells: Possible mediation by decreased JNK/AP-1 signaling. J Nutr Biochem 20: 663-676, 2009.

38. Lee SJ, Park SS, Lee US, Kim WJ and Moon SK: Signaling pathway for TNF-alpha-induced MMP-9 expression: Mediation through p38 MAP kinase and inhibition by anti-cancer molecule magnolol in human urinary bladder cancer 5637 cells. Int Immunopharmacol 8: 1821-1826, 2008.

39. Wang WH, Hullinger RL and Andrisani OM: Hepatitis B virus $X$ protein via the $\mathrm{p} 38 \mathrm{MAPK}$ pathway induces $\mathrm{E} 2 \mathrm{~F} 1$ release and ATR kinase activation mediating p53 apoptosis. J Biol Chem 283: 25455-25467, 2008.

40. Kim MS, Lee EJ, Kim HR and Moon A: p38 kinase is a key signaling molecule for H-Ras-induced cell motility and invasive phenotype in human breast epithelial cells. Cancer Res 63: 5454-5461, 2003

41. Bernhard EJ, Gruber SB and Muschel RJ: Direct evidence linking expression of matrix metalloproteinase $9(92-\mathrm{kDa}$ gelatinase/collagenase) to the metastatic phenotype in transformed rat embryo cells. Proc Natl Acad Sci USA 91: 4293-4297, 1994. 